\title{
Coordenação da qualidade: proposta de estrutura e método para cadeias de produção agroalimentares
}

\author{
Miguel Angel Aires Borrás \\ José Carlos de Toledo \\ UFSCar
}

\begin{abstract}
Resumo
A realidade competitiva vivenciada pelas empresas desestimula que elas ajam de forma isolada, requerendo ações coordenadas com outras empresas da mesma cadeia. Por essa razão, a busca pela coordenação de cadeias de produção visando à melhoria da qualidade do produto e à redução dos custos associados às perdas da produção e aos custos de transação está se tornando uma prática mais comumente adotada por empresas que partilham o objetivo de adicionar valor e competitividade ao produto final da cadeia. Este artigo aborda o problema da coordenação da qualidade em cadeias de produção agroalimentares (CPAs). Com base em conceitos da revisão bibliográfica e de evidências empíricas, são propostos uma Estrutura (ECQ - Estrutura para Coordenação da Qualidade) e um Método (MCQ - Método para Coordenação da Qualidade) para a coordenação da qualidade em CPAs. Em algumas cadeias, a implementação da ECQ/MCQ vem sendo ilustrada bem como usada como alternativa técnica possível e confiável para coordenar e garantir a qualidade do produto final.
\end{abstract}

Palavras-chave

Coordenação da qualidade, segurança do alimento, cadeia de produção agro-alimentar, gestão da qualidade.

\section{Quality coordination: a proposal of structure and method for agri-food production chains}

\begin{abstract}
Today's reality prevents companies in general from planning or acting alone, requiring that they engage in coordinated action with others in the same production chain. For this reason, production chain coordination to improve product quality and reduction of production wastes and transactions costs is becoming a widespread practice to promote integrated management of companies that share the goal of adding value to the chain's final product. This paper discusses the problem of quality in agri-food supply chains (ASC). Based on wide bibliography review, a structure (ECQ) and a method (MCQ) are proposed for the quality coordination in ASC. In some chains the ECQ/MCQ implementation has been studied and used as a possible and liable technical alternative to coordinate and assure the final product quality.
\end{abstract}

Key words

Quality coordination, safety product, agri-food supply chain, quality management. 


\section{INTRODUC̣̃̃O}

A crescente preocupação com a segurança e a qualidade dos alimentos, como um dos principais fatores competitivos das cadeias de produção agro-alimentares (CPAs), exige que estas busquem mecanismos para melhoria da gestão da qualidade.

De um lado, para dar evidência à qualidade de seus produtos, garantindo que estes tenham as qualidades intrínsecas esperadas pelo consumidor e incrementando a qualidade percebida pelo mercado. De outro lado, melhorando a qualidade de conformação, buscando reduzir custos de falhas e de perdas ao longo da cadeia.

São vários os exemplos de registros de problemas econômicos e de saúde pública decorrentes da produção e consumo de alimentos contaminados em todo o mundo. Dentre os mais recentes e conhecidos problemas desse tipo, no setor agroindustrial, pode-se citar o mal da "vaca louca" (encefalopatia espongiforme bovina ou bovine spongiform encephalopathy - BSE).

adotarem práticas de gestão que melhor garantissem a qualidade e segurança da carne produzida, como, por exemplo, a adoção da prática de identificação e rastreabilidade (HEIM; MUMFORD, 2005).

A qualidade do produto final, bem como a eficiência da CPA em termos de desperdícios e de custos com perdas e retrabalhos, dependem de ações e práticas coordenadas entre segmentos e agentes e das transações de bens, serviços e informações na cadeia. Nesse contexto, evidencia-se a importância de gerenciar a qualidade de maneira coordenada ao longo das CPAs.

Os instrumentos tradicionais de gestão da qualidade se limitam a ações no âmbito das empresas, e observa-se a necessidade do desenvolvimento de métodos e ferramentas de apoio à coordenação da qualidade na cadeia como um todo, capacitando os agentes da cadeia a definir, receber, processar, difundir e utilizar informações de modo a implantar e gerenciar as estratégias da qualidade.

Este artigo tem como objetivo apresentar um modelo de gestão, desenvolvido a partir de evidências práticas e teóricas, constituído por uma estrutura e por um método para coordenação da qualidade, que auxilie nos processos de garantia e de melhoria da qualidade em CPAs.

Ainda que se reconheça a influência das características das transações e dos agentes no processo de coordenação de uma cadeia produtiva, a consideração de tais aspectos

O problema do mal da "vaca louca" teve sua origem na primeira metade da década de 1970, quando parte do rebanho britânico de gado bovino passou a ser tratado com dieta à base de carne e osso (RAMASAMY, 2004; HEIM; MUMFORD, 2005).

Porém, o primeiro caso registrado de contaminação com o agente da BSE surgiu em 1985, na Grã-Bretanha, tendo atingido seu ápice na década de 1990 (HEIM; MUMFORD, 2005).

Em 1996, provou-se cientificamente que o mal da "vaca louca" poderia ser transmitido aos seres humanos através do consumo de carne contaminada pelo agente da BSE, causando a doença de Creutzfeldt-Jakob (BERG, 2004). Iniciado na Grã-Bretanha, em 2002 o mal da "vaca louca" já atingia toda a Europa, os EUA, o Japão e Israel (HEIM; MUMFORD, 2005).

Percebe-se, então, que a utilização de uma dieta contaminada com o agente da BSE na etapa de produção agropecuária gerou perdas para toda a cadeia de produção da carne de gado bovino e para as nações que abrigaram focos da doença, gerando perdas econômicas significativas e a desconfiança do consumidor, que passou a exigir maior segurança da carne consumida (BERG, 2004). Tal reação do mercado obrigou as cadeias de carne bovina, espalhadas em todo o mundo, a encontra-se fora do escopo deste artigo, o qual se limita a uma proposta de auxílio à melhoria da competitividade das cadeias, por meio da coordenação das práticas de gestão e de melhoria da qualidade realizadas por seus agentes.

$\mathrm{O}$ modelo apresentado é genérico para CPAs, devendo ser entendido como um modelo de referência que deve ser adaptado às especificidades de cada cadeia.

\section{REFERENCIAL TEÓRICO}

Segundo Boehlje et al. (1998), a motivação para coordenação de cadeias, a fim de ganhar vantagem competitiva, se dá em três fases seqüenciais em busca de: a) melhoria na eficiência e redução de custos; b) redução de riscos quanto à qualidade, quantidade e segurança do alimento; e c) satisfação das necessidades dos consumidores. Em todas essas motivações pode-se dizer que há a presença de algum aspecto da qualidade.

Assim, pode-se afirmar que coordenar a qualidade ao longo de uma cadeia de produção implica em gerenciar informações referentes aos requisitos exigidos da qualidade do produto e da gestão da qualidade e ao grau de atendimento destes pelos agentes da cadeia. Tal gerenciamento deve ser estabelecido tanto para os agentes quanto 
para toda a cadeia, buscando a integração de todos os seus segmentos.

De fato, para Frohlich e Westbrook (2001) quanto mais amplo o grau de integração da cadeia de produção, melhores serão os seus indicadores de desempenho.

Além disso, Schiefer (2002) afirma que a gestão da qualidade na cadeia de produção diz respeito ao fluxo de informações sobre as características de produção, as características da qualidade, o controle do produto e dos processos e sobre o suporte a atividades de melhoria da qualidade.

Assim, neste artigo define-se coordenação da qualidade em cadeias de produção como o conjunto de atividades planejadas e controladas por um agente coordenador, tendo por finalidade aprimorar a gestão da qualidade e auxiliar nos processos de garantia e melhoria da qualidade dos produtos ao longo da cadeia, por meio de um processo de aquisição, gestão e distribuição de informações, contribuindo para a melhoria da satisfação dos clientes e para a redução de custos e perdas na cadeia.

Baines e Davies (1998) e Ziggers e Trienekens (1999) listaram alguns dos resultados que podem ser alcançados com a garantia da qualidade numa CPA: a) aumento da probabilidade de fornecer produtos de qualidade por monitoramento, ação corretiva e melhoria contínua; b) habilidade de responder e controlar situações de emergência; c) habilidade para responder a requisitos de órgãos públicos e de consumidores; d) aumento da confiança do consumidor com relação a toda a cadeia; e) adição de valor ao produto; e f) redução de custos totais da produção nos segmentos da cadeia.

A seção seguinte do artigo descreve a proposta da Estrutura e do Método para Coordenação da Qualidade (ECQ e MCQ).

\section{METODOLOGIA DE PESQUISA}

A pesquisa descrita neste artigo teve como objetivo principal o desenvolvimento de uma proposta de método para coordenação da qualidade (MCQ), baseada numa revisão bibliográfica e na consulta a empresários e especialistas do setor. Essa pesquisa pode ser classificada como básica/ teórica quanto ao método utilizado, exploratória e descritiva quanto ao seu objetivo.

A pesquisa foi desenvolvida em três etapas:

1) Revisão bibliográfica: etapa mais longa da pesquisa; os dados e informações nela coletados serviram de base para as outras etapas da pesquisa. Como fontes de dados e informações foram utilizados livros, artigos científicos, revistas, anais de congressos, leis e normas, páginas de Internet, visitas técnicas e entrevistas informais com especialistas. Devido ao caráter inovador da presente pesquisa, houve a necessidade de se pesquisar uma diversidade de temas: definições de qualidade, alimentos e assuntos afins; teorias de troca; teoria acerca da gestão de cadeias de produção; métodos de pesquisa; teorias de marketing; métodos e ferramentas da qualidade e outras teorias econômicas e de gestão, conforme Borrás (2005);

2) Elaboração do Método para Coordenação da Qualidade: Nesta etapa, com base nos dados e informações levantados na etapa 1 , definiu-se o conceito de coordenação da qualidade, a Estrutura para Coordenação da Qualidade (ECQ) e a lógica de implantação e aplicação do Método para Coordenação da Qualidade (MCQ), além de se propor um tipo de estrutura para o agente coordenador; e

3) Validação e Ilustração: com o intuito de verificar se a ECQ e o MCQ são aplicáveis na prática, validou-se a ECQ e o MCQ por meio de um workshop realizado com 9 representantes de média gerência de 3 usinas sucro-alcooleiras e 4 produtores rurais fornecedores de cana-de-açúcar para as referidas usinas, localizadas na região de Sertãozinho-SP. De cada usina, reuniram-se representantes do departamento agrícola, do setor de produção e do setor de vendas, estes últimos tendo sido escolhidos para trazer à discussão a problemática do fornecimento dos açúcares granulado, invertido e líquido para outros segmentos desta cadeia agroalimentar. O workshop teve duração de 5 horas, e foi dividido em três partes: a) apresentação da ECQ e do MCQ, com duração de 1 hora; b) discussão da ECQ e do MCQ, com duração de 3 horas e c) preenchimento de questionário sobre a ECQ e o MCQ e discussão dos resultados, com duração de 1 hora. Neste artigo, o resultado do workshop apresenta-se na seção de validação do MCQ. Além desta avaliação, foram elaboradas duas ilustrações de aplicação da ECQ e do MCQ: uma para a cadeia de produção agroindustrial da maçã e outra para a cadeia de produção agroindustrial do queijo meia-cura.

A escolha de empresas do setor sucro-alcooleiro para a validação se deu por dois motivos: facilidade para reunir representantes de empresas líderes de mercado desta cadeia de produção e importância estratégica da indústria sucroalcooleira.

Todas as usinas escolhidas são gerenciadas com uma visão sistêmica da cadeia do açúcar, comprovada por visitas técnicas e entrevistas com gerentes dessas usinas, antes da execução do workshop.

Além disso, todas elas são certificadas pela ISO, possuindo os certificados ISO 9001:2000 e ISO 14000, além de já possuírem os sistemas de Análise de Perigos e Pontos Críticos de Controle (APPCC), Boas Práticas Agrícolas (BPA) e Boas Práticas de Higiene (BPH), implantados ou em fase de implantação em suas linhas de produção de açúcar. 
Todos os gerentes presentes no workshop haviam participado ou estavam participando dos processos de implantação e auditoria interna relativos a tais certificações. Essas características das usinas e dos gerentes foram importantes para garantir que todos possuíssem um nível mínimo de conhecimento e aplicação de sistemas da qualidade.

Havia entre os participantes um mínimo de reconhecimento de que a coordenação da qualidade na cadeia seria o próximo passo na busca de mais elevados patamares de capacidade competitiva.

Todos os gerentes que participaram do workshop tinham ao menos dez anos de experiência no cargo, com profundo conhecimento das práticas de produção aplicadas em suas respectivas áreas de atuação: agrícola, produção e vendas/ expedição.

A ausência de representantes do segmento de distribuição e de outras empresas compradoras de açúcar para elaboração de outros produtos alimentícios foi satisfatoriamente coberta pela presença dos gerentes de vendas das usinas, que possuíam forte relacionamento e conhecimento sobre os segmentos a jusante na cadeia.

Os produtores rurais escolhidos são graduados em administração ou em engenharia agronômica. Para uma validação confiável da ECQ e do MCQ, o entendimento dos princípios teóricos e da lógica de funcionamento e implantação do MCQ, era de fundamental importância para a pesquisa.

\section{A ESTRUTURA, O MÉTODO E O AGENTE PARA COORDENAC̄̃̃O}

A ECQ apresenta as funções básicas de: a) identificar e desdobrar os requisitos da qualidade do produto, para satisfazer a qualidade demandada pelo mercado e pelo ambiente institucional; e b) definir, implementar e controlar processos de melhoria da qualidade do produto e de gestão da qualidade.

São quatro os elementos que formam a ECQ: a) a CPA, seus segmentos e agentes; b) os requisitos da qualidade do produto e da gestão da qualidade; c) o Método de Coordenação da Qualidade; e d) o agente coordenador (Figura 1).

Nas seções seguintes são caracterizados os elementos constituintes da ECQ listados anteriormente e cuja interrelação é mostrada na Figura 1.

\section{Requisitos da qualidade do produto e da gestão da qualidade}

O não atendimento aos requisitos da qualidade do produto (RQP) e da gestão da qualidade (RGQ) pode resultar na per-

Figura 1: Elementos da Estrutura para Coordenação da Qualidade (ECQ).

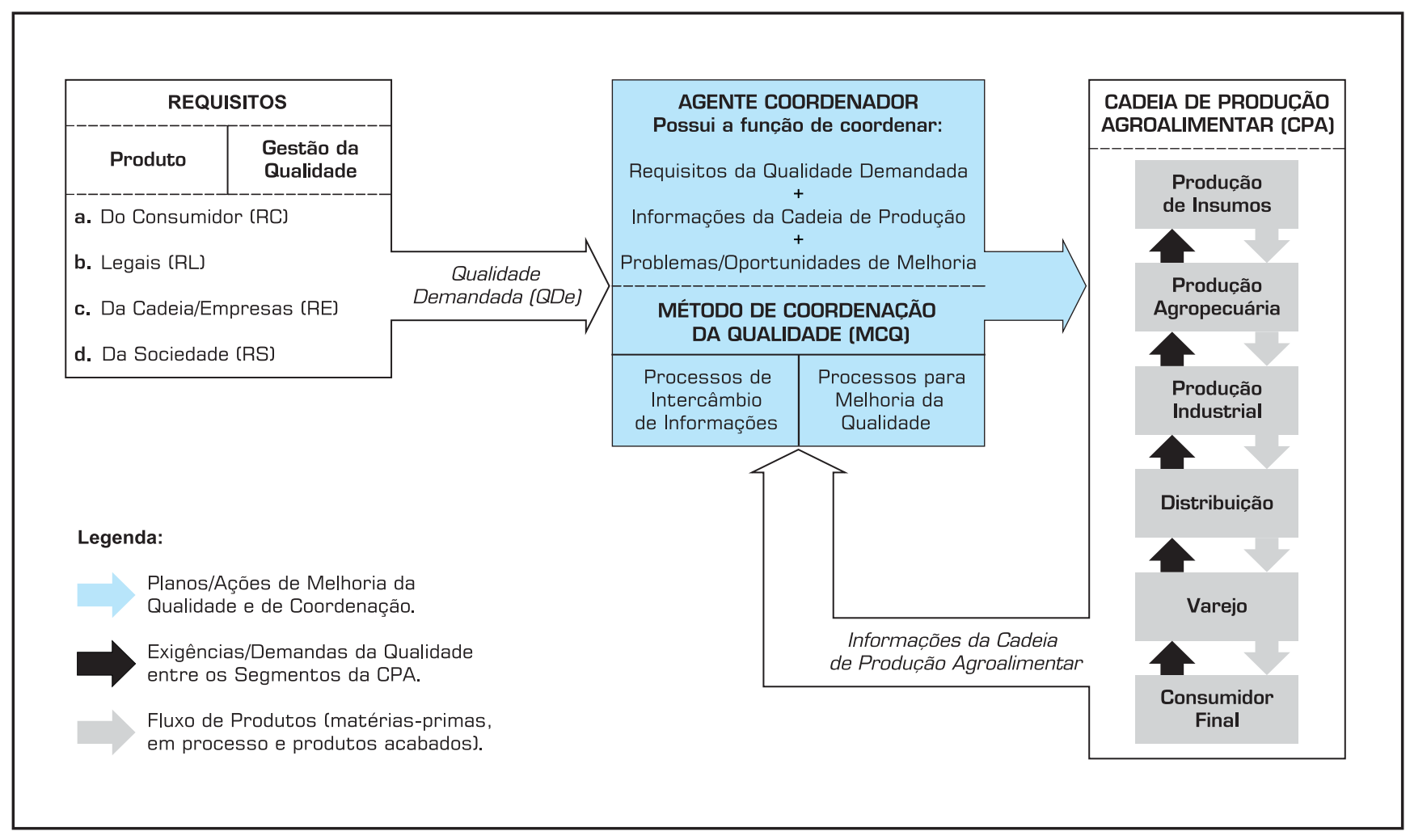


da de competitividade da cadeia, uma vez que o seu produto não estaria atendendo da melhor maneira possível o que é exigido pelo mercado, incentivando-o a procurar produtos de outra CPA. Garantir que os requisitos sejam atendidos é o principal objetivo da ECQ/MCQ.

Os RQP podem ser traduzidos como sendo o conjunto de requisitos válidos para o produto, ou seja, padrões de qualidade do produto que, ao serem atendidos, seriam capazes de satisfazer ao máximo as necessidades e expectativas dos agentes, das regulamentações e do consumidor final. Já os RGQ podem ser descritos como o conjunto de requisitos necessários para planejar, executar e controlar atividades que buscam garantir que os projetos, processos, produtos e serviços elaborados pela CPA satisfaçam, ao máximo, as necessidades e expectativas do consumidor final e de seus próprios agentes. Os RGQ dizem respeito às práticas que os agentes da CPA podem adotar, tais como Identificação e Rastreabilidade de produtos, Boas Práticas Agrícolas e de Fabricação, etc. Por sua vez, a Qualidade Demandada (QDe), ponto de partida para a aplicação da ECQ/MCQ, é composta por Requisitos Legais (RL), Requisitos do Consumidor (RC), Requisitos da Cadeia/Empresas (RE) e Requisitos da Sociedade (RS), cujas definições são mostradas no Quadro 1.

$\mathrm{Na}$ seção seguinte é abordado como possíveis balanceamentos e soluções de compromisso são efetuados entre tais requisitos. A QDe deve refletir os aspectos contratuais e mercadológicos internos e externos à cadeia, abrangendo as necessidades e as expectativas explícitas e implícitas dos consumidores finais, clientes e do ambiente institucional.

\section{O Método para coordenação da qualidade (MCQ)}

$O$ instrumento que auxilia no processo de coordenação da qualidade é denominado Método para Coordenação da Qua- lidade e deve ser capaz de: a) coordenar o fluxo de receber, armazenar e enviar informações entre os agentes da CPA e o agente coordenador; b) receber e armazenar informações do ambiente institucional; c) tratar as informações recebidas e gerar diagnóstico a respeito da qualidade de produto e de processo praticada pela CPA; d) disponibilizar informações que possibilitem a tomada de decisões do agente coordenador; e) capacitar o agente coordenador para a elaboração de planos de ação e controle destes, quando implantados com os agentes da CPA; f) proporcionar visão holística das ações tomadas pelos agentes da $\mathrm{CPA}$, quanto às práticas de gestão da qualidade.

Em outras palavras, o MCQ busca auxiliar o agente coordenador a organizar, processar e analisar informações sobre a qualidade dos produtos e sobre a gestão da qualidade praticada pelos agentes dos segmentos da cadeia, e estabelecer um fluxo de informações, entre o agente coordenador e as empresas, com o intuito de possibilitar um ciclo contínuo de melhoria. O MCQ proposto é formado por oito módulos:

O módulo 1 tem por principal função identificar e agregar os requisitos da sociedade (RS), legais (RL), do consumidor (RC), da cadeia de produção e empresas que a compõem (RE) e transformá-los em uma "lista" de requisitos demandados. A essa lista é dado o nome de Qualidade Demandada (QDe).

No caso dos requisitos contraditórios, a lógica do desdobramento por meio da ferramenta QFD (Quality Function Deployment) possibilita eliminar tais contradições na medida em que impede a continuidade daquele que agrega menos valor ao produto ou na medida em que força o encontro de um terceiro requisito que seja o meio-termo entre os outros dois.

Há de se obedecer uma escala de prioridades para fazer tal filtragem. Se houver duplicidade entre um RL e um RE,

Quadro 1: Tipos de requisitos da qualidade.

\begin{tabular}{|l|l|}
\hline \multicolumn{1}{|c|}{ REQUISITOS DA QUALIDADE } & \multicolumn{1}{c|}{ DEFINIÇÃo } \\
\hline Requisitos Legais $(R L)$ & $\begin{array}{l}\text { Conjunto de normas, regulamentos, códigos e procedimentos formalizados } \\
\text { por legislação e que possam influenciar ou definir as características da } \\
\text { qualidade de um produto. }\end{array}$ \\
\hline Requisitos do Consumidor (RC) & $\begin{array}{l}\text { Consistem nos desejos e expectativas em relação a um determinado produto } \\
\text { a ser entregue ou serviço a ser prestado por um fornecedor. }\end{array}$ \\
\hline Requisitos da Cadeia/Empresas (RE) & $\begin{array}{l}\text { Expressam as necessidades ou prioridades das cadeias/empresas, } \\
\text { explicitadas em termos quantitativos ou qualitativos, objetivando definir } \\
\text { características que o produto deve conter, alinhadas às estratégias } \\
\text { competitivas e de imagem da empresa e da cadeia. }\end{array}$ \\
\hline Requisitos da Sociedade (RS) & $\begin{array}{l}\text { Conjunto de normas, regulamentos, códigos, procedimentos, fatores de } \\
\text { saúde, de segurança, do meio ambiente e de conservação de energia, } \\
\text { formalizados por legislação ou praticados como valores socioculturais. }\end{array}$ \\
\hline
\end{tabular}


por exemplo, deve-se eliminar o RE, pois o RL é de cumprimento obrigatório. Em outras palavras, deve-se eliminar o requisito de menor prioridade na escala que obedece a seguinte ordem decrescente de prioridade: Requisito Legal (RL), Requisito do Consumidor (RC), Requisito da Empresa (RE) e Requisito da Sociedade (RS).

O grau de prioridade anteriormente mostrado foi validado pelos gerentes e produtores rurais presentes no workshop para avaliação da ECQ/MCQ, ainda que tenha ficado claro que, em muitos casos, há uma sobreposição dos diferentes tipos de requisitos.

Observa-se que tal ordenamento por prioridades dos requisitos tem validade nos dias atuais e num futuro de médio prazo. A maior exigência dos clientes, o incremento do rigor da legislação no que tange ao comércio nacional e internacional, a maior preocupação com o meio ambiente e o acirramento da competitividade no setor alimentício farão com que todos os requisitos estejam num mesmo patamar de prioridade, tornando a gestão dos mesmos ainda mais complexa.

Em resumo, o módulo 1 do MCQ é composto, basicamente, por bancos de dados e seu funcionamento se resumiria a três fases: coleta de dados e informações junto às fontes de informação, filtragem e envio das QDe ao módulo 2 do MCQ.

O módulo 2 é executado em duas etapas. A primeira tem a função de desdobrar a QDe nos requisitos de qualidade de produto (RQP) e de gestão da qualidade (RGQ). Em outras palavras, significa identificar os "o quês" a serem buscados pela CPA e "como" esses "o quês" devem ser alcançados e gerenciados. A segunda etapa deste módulo tem a função de identificar e armazenar em diferentes bancos de dados, os "o quês" e "comos" a serem empregados em cada segmento da CPA e que foram gerados na etapa 1. Uma ferramenta que a ser utilizada neste módulo é o Desdobramento da Função Qualidade, ou Quality Function Deployment (QFD). Busca-se que o resultado do módulo 2, conjunto de "o quês" e de "comos" fundamentais, seja comunicado a cada segmento responsável por cumpri-los.

O módulo 3 consiste num "módulo de ajuste" que tem como função eliminar incompatibilidades entre os "o quês" e "comos" identificados no módulo anterior. Após o envio do resultado do módulo 2 para os segmentos da cadeia de produção, estes devem avaliá-los e indicar quais os válidos e aqueles que não são factíveis de realização, seja por problemas financei-

Além disso, também é função deste módulo, verificar se existem requisitos conflitantes ou se um engloba algum outro. No caso de requisitos idênticos, elimina-se a duplicidade, e em caso de requisitos que abrangem outros, mantém-se o mais abrangente. Dos requisitos dessa lista, definem-se quais são os requisitos mais significativos ou características-chave que serão objetos de coordenação e que, assim, deverão ser desdobradas no módulo 2 .

As características-chave ou significativas do produto são tópicos funcionalmente importantes, características de engenharia, críticos para a função e para a qualidade. São requisitos que apresentam um impacto significativo no custo, desempenho e segurança do produto final, sendo que somente tais requisitos-chave serão enviados ao módulo de desdobramento da qualidade, no MCQ. A lista desses requisitos-chave dá origem ao requisito da qualidade ou qualidade demandada (QDe).

Por fim, é também neste módulo que são medidos os indicadores de desempenho da CPA, para posterior reavaliação no módulo 8. A base tecnológica deste módulo seria composta de ferramentas e métodos para pesquisa de mercado, além de mecanismos para obtenção e organização de dados gerenciais. A Figura 2 resume as funções do módulo 1 do Método para Coordenação da Qualidade (MCQ). ros, técnicos, de pessoal, etc. Se necessário, os segmentos da CPA podem modificar ou acrescentar os "o quês" e "comos" que forem necessários.

A eliminação ou acréscimo de certos "o quês" e "como's", por um determinado segmento da CPA, pode causar problemas de conformidade para um segmento anterior ou posterior a esse. Logo, é função do módulo 3 indicar os conflitos ao agente coordenador e auxiliá-lo nos contatos com as empresas dos segmentos envolvidos, em busca de um acordo. Após o ajuste, os resultados são enviados aos segmentos responsáveis por cada conjunto de "o quês" e "comos" para nova avaliação, repetindo-se este processo até se conseguir um conjunto ajustado de "o quês" e "comos" ao longo da cadeia.

Portanto, o resultado do módulo 3 indica qual deve ser o "caminho correto" para satisfazer a QDe, ou seja, indica o quê deve ser buscado e como isso pode ser alcançado pelas empresas de cada segmento da CPA.

No entanto, esse "caminho correto" já pode estar sendo praticado ao longo da cadeia. Cabe, então, ao módulo 4, verificar se os requisitos de qualidade de produto e de gestão da qualidade, já utilizados por cada segmento da cadeia de produção, correspondem ao "caminho correto" indicado pelo módulo 3. Essa verificação é realizada confrontando 
as características de produto e de gestão que seriam ideais e indicadas pelo MCQ com as características equivalentes, que estão sendo praticadas no momento pelas empresas da CPA.

No caso de alguma característica, na prática, apresentar diferença em relação ao ideal, indicaria a existência de um desvio, ou de requisito de produto ou de gestão da qualidade. Tanto o módulo 3 quanto o módulo 4 são constituídos basicamente por bancos de dados e, nessas etapas, o agente coordenador e as empresas da CPA exerceriam uma importante e fundamental função de análise e geração de dados e informações.

Figura 2: Resumo das funções do módulo 1 do MCQ.

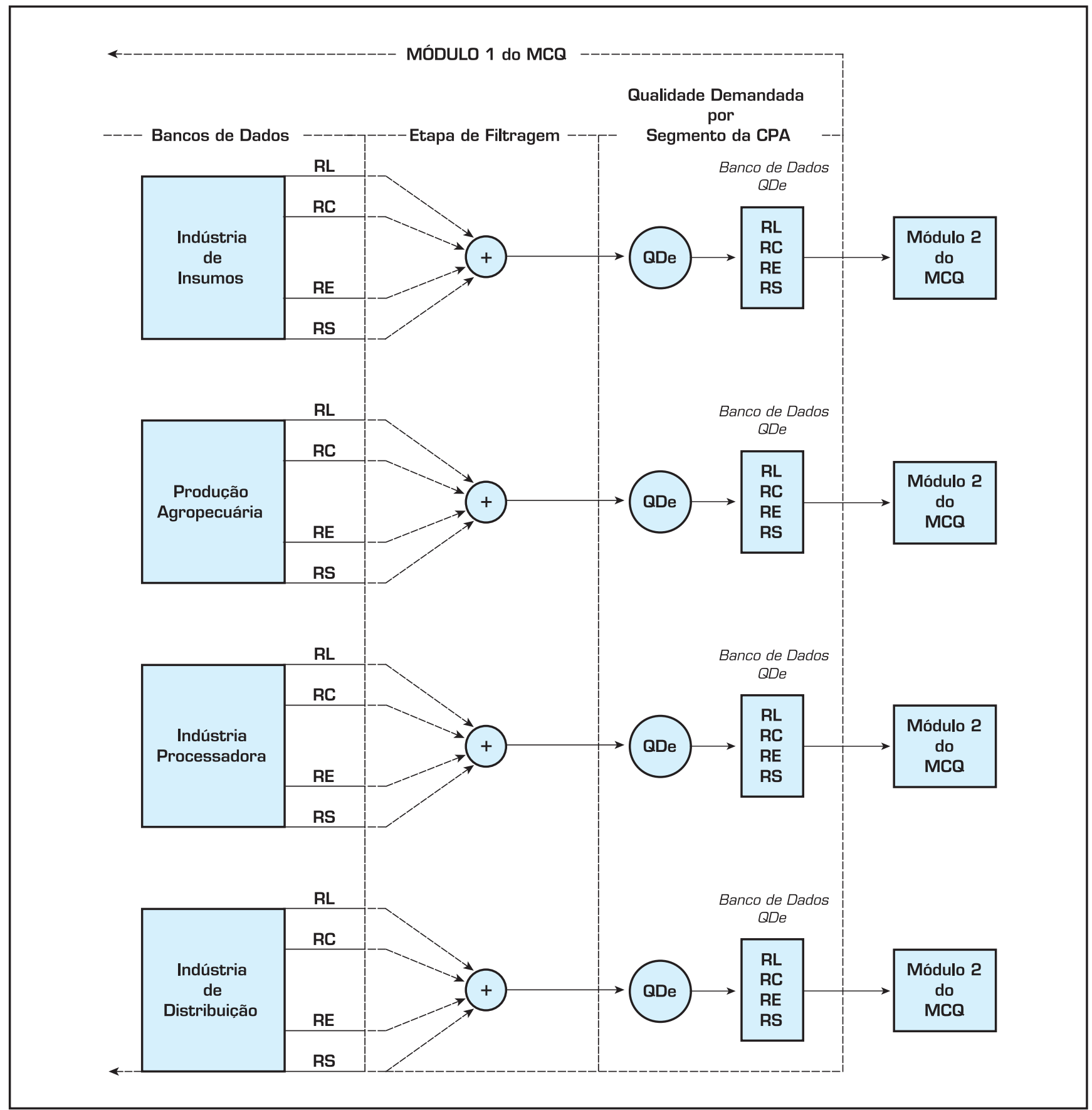

Fonte: Borrás (2005). 
O módulo 5 tem por função "listar" os desvios identificados no módulo 4, identificar suas causas e os segmentos responsáveis por elas, e avaliar os itens de verificação e de controle das etapas críticas de produção para cada segmento da CPA. Após essa medição, passa-se a informação gerada ao módulo 6 (Figura 3).

O módulo 5 é constituído por bancos de dados e faz uso de ferramentas da qualidade como os diagramas de Causae-Efeito, de Relações e de Afinidades.

O módulo 6 tem por função analisar as causas dos desvios identificados no módulo 4 e listados no módulo 5, e propor planos de ação para a eliminação de tais desvios e de suas causas. Essa análise e geração de planos de melhoria são feitos a partir do uso de métodos e ferramentas da qualidade, aplicados junto aos segmentos causadores dos desvios.

Já o módulo 7 tem por função comunicar os resultados do módulo 6 aos segmentos da CPA, além de executar as funções de controle da implantação dos planos de melhoria da qualidade, pelos segmentos da cadeia envolvidos (Figura 4).

A avaliação da eficácia dos planos de ação é feita com nova medição dos índices de desempenho da cadeia e de sua confrontação com a primeira medição realizada durante execução do módulo 1, para corroborar os planos

Figura 3: Lógica de funcionamento do módulo 5 do MCQ.

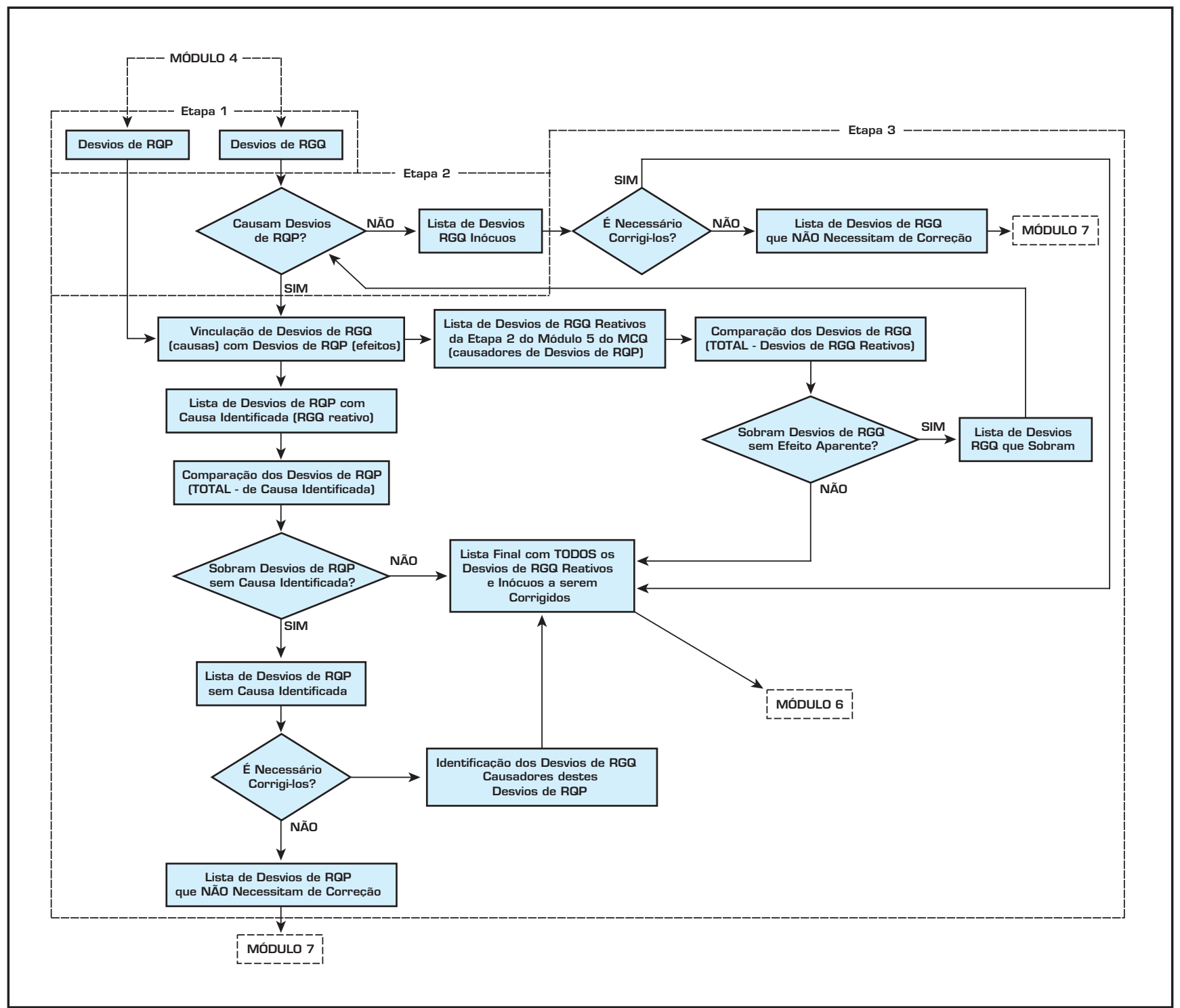

Fonte: Borrás (2005). 
propostos ou para transformá-los em processos operacionais padrão.

Se durante tal avaliação forem observadas anomalias, ou seja, variabilidade indesejável nos índices de desempenho da CPA, deve-se identificar quais são as possíveis causas responsáveis por essa variabilidade, reiniciando a aplicação dos módulos 6 a 7 . Para isso poderia ser utilizado o método PDCA/MASP de análise e solução de problemas.

Finalmente, é função do módulo 8 a autoavaliação do próprio método de coordenação. Periodicamente, indicadores de desempenho definidos para o MCQ são medidos, sendo passíveis de aprovação ou não. Se houver a plena aprovação de tais fatores, ou seja, se para todos os fatores a resposta for "sim", o MCQ segue sendo utilizado normalmente, caso contrário, o MCQ é analisado buscando descobrir as falhas que devem ser sanadas, de modo que o método seja aperfeiçoado até atingir a plenitude de aprovação em sua auto-avaliação.

Os módulos do MCQ podem ser classificados como executores de Processos de Intercâmbio de Informações (PII) ou como executores de Processos para Melhoria da Qualidade (PMQ). Os PII, que são os módulos 2 e 7 do MCQ, têm a função principal de estabelecer o intercâmbio contínuo de informações entre o agente coordenador e os agentes dos segmentos da CPA. Tais módulos capturam, transformam e transmitem dados e informações entre todos os elementos constituintes da ECQ.

Os demais módulos do MCQ, ou seja, os módulos 2 a 6 e o módulo 8, têm a função de servir como instrumento de apoio, para que o agente coordenador e os agentes da CPA possam analisar e utilizar as informações intercambiadas entre os elementos da ECQ.

Esse intercâmbio de dados e informações permite a geração de planos de ação para melhoria da qualidade do produto e de gestão da qualidade a ser implementados pelos agentes da CPA e controlados pelos mesmos e pelo agente coordenador, tendo a finalidade de reduzir perdas e custos de produção, garantir a qualidade do produto final e incrementar a competitividade de toda a cadeia produtiva. A composição dos recursos tecnológicos (programas computacionais, bancos de dados, sistemas de informação gerenciais, etc.) do MCQ pouco varia de um módulo para outro, mas o grau de utilização desses recursos determina a diferença entre quais seriam os componentes tecnológicos fundamentais para os módulos executores dos PII e para os executores dos PMQ.

\section{0 agente coordenador: estrutura e funções}

Segundo Zylbersztajn (1995), como os sistemas agroindustriais são sujeitos a choques e pressões que requerem respostas coordenadas, espera-se que as estruturas de governança híbrida (contrato) ou hierárquica (integração vertical) sejam as mais adotadas nestes sistemas. Porém, para os casos em que haja necessidade de ajustamentos de coordenação estrita para adaptação das cadeias de produção, envolvendo grande número de agentes, motiva-se o surgimento de organizações para realizar o papel de agente coordenador (ZYLBERSZTAJN, 1995).

\section{MCQ tem a função básica de auxiliar o agente coordenador a gerenciar a dade praticada na cadeia.}

Na Estrutura para Coordenação da Qualidade (ECQ), o agente coordenador poderia ser: uma empresa de um segmento da própria cadeia, um grupo de profissionais constituído por representantes de cada segmento da CPA, uma empresa independente organizada e contratada para exercer tal função, uma instituição governamental, ou uma associação representativa da cadeia de produção em questão. Como o Método para Coordenação da Qualidade (MCQ) é um instrumento de apoio à função de gerenciamento do agente coordenador, cabe a ressalva de que é fundamental a definição de uma adequada estrutura de governança para o agente coordenador, para que se consiga integrar o MCQ, o agente coordenador e a CPA. As funções do agente coordenador podem ser descritas como:

a) Gerenciamento do Sistema de Informações: espera-se que o Agente coordenador gerencie o sistema de informações no que tange a: 1) Requisitos da Qualidade do Produto (RQP) final; 2) Requisitos da Qualidade do Produto para cada segmento da cadeia de produção; 3) Requisitos de Gestão da Qualidade; 4) Situação atual do atendimento dos RQP; 5) Situação atual da aplicação das práticas de Gestão da Qualidade; e 6) Indicadores de desempenho da cadeia e de cada segmento;

b) Identificação e Comunicação de Problemas e Oportunidades de Melhoria: o agente coordenador, com base nas informações que gerencia (vide acima), deve identificar a ocorrência de desvios (problemas) e, além disso, identificar oportunidades de melhoraria. Mas não basta identificá-los, é fundamental compartilhar isso com todos os segmentos da cadeia, de modo a sinalizar para cada um deles onde há problemas e onde há possibilidade de investir em melhorias;

c) Análise de Problemas e Soluções: espera-se que o agente coordenador organize reuniões periódicas com os representantes de cada segmento da cadeia de produção, nas quais, a partir da priorização e do detalhamento 
Figura 4: Lógica de funcionamento dos módulos 6 e 7 do MCQ.

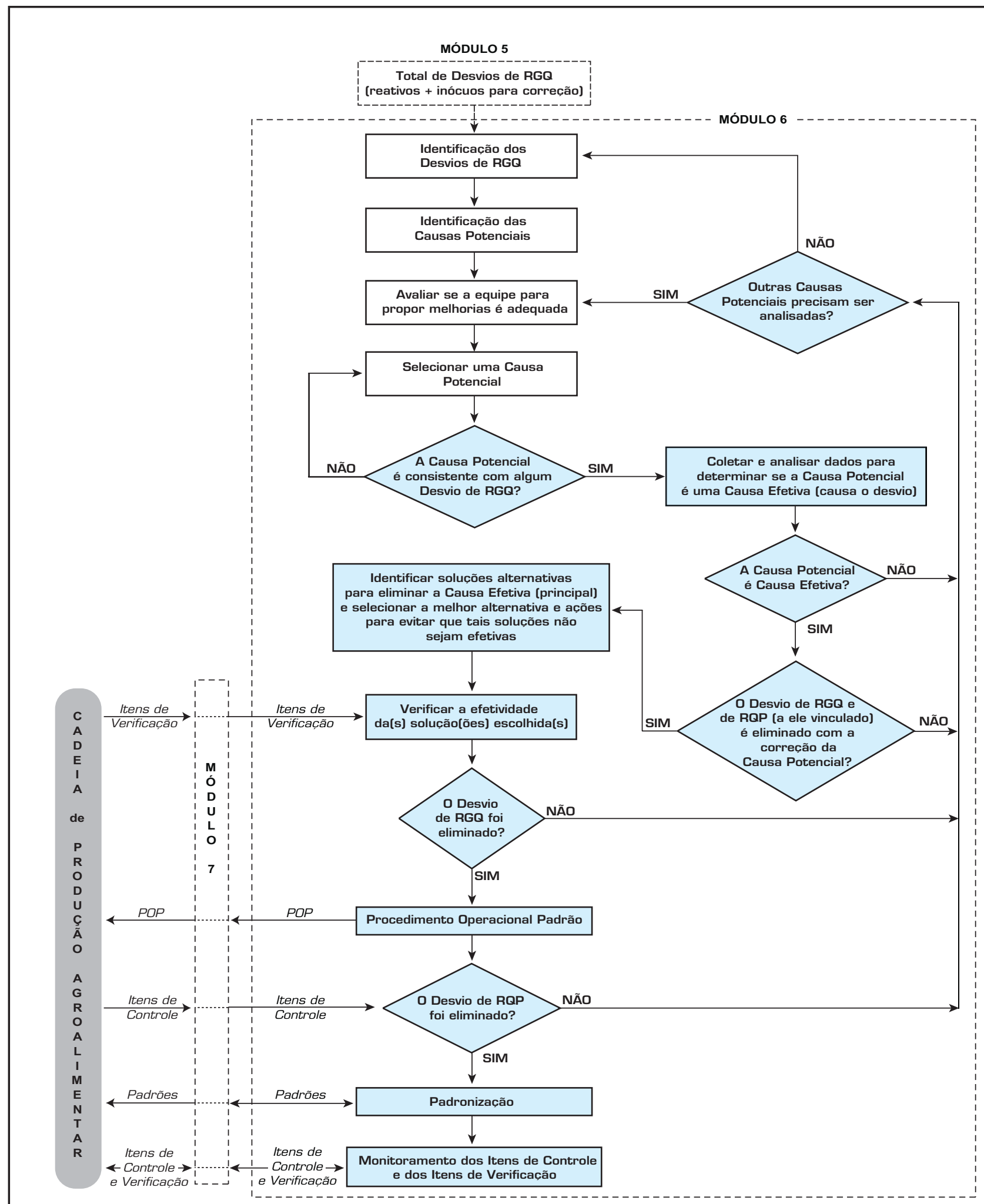

Fonte: Borrás (2005) 
dos problemas identificados, são analisadas as causas fundamentais dos problemas e também são planejadas, em conjunto, ações de melhoria tanto para atuar nessas causas, buscando soluções de tais problemas, como para aproveitar as oportunidades de melhoria levantadas; e

d) Acompanhamentos: mais do que simplesmente propor ações de melhoria, é fundamental que estas sejam efetivamente implantadas e acompanhadas.

Assim, é preciso que o Agente coordenador viabilize meios de acompanhar a implantação das ações propostas, verificando se os resultados esperados estão sendo obtidos e, também, se tais ações não estão causando efeitos colaterais nas empresas dos segmentos ou em toda cadeia de produção. A Figura 5 resume as funções que o agente coordenador desempenharia na estrutura para a coordenação proposta.

Na seção seguinte o Procedimento de Implantação da ECQ/MCQ (PIEM) é sucintamente descrito.

\section{ATIVIDADES PARA IMPLANTAC̣ÃO DA ECQ E DO MCQ}

Propõem-se as seguintes atividades subseqüentes como condições necessárias para a implantação da ECQ/MCQ numa CPA: 1) compreender a ECQ e seus elementos (o que inclui o método para coordenação), ou seja, os agentes pertencentes à cadeia ou ligados a ela devem entender a importância da ECQ, os objetivos, bem como sua dinâmica de funcionamento na $\mathrm{CPA}$; 2) definir e organizar o agente co- ordenador, identificando sua estrutura e seu perfil de atuação. É ele quem vai liderar as atividades de coordenação da qualidade na CPA, e sua indicação adequada é fundamental para o sucesso da implantação da ECQ; 3 ) efetuar um diagnóstico da CPA, visando analisar as necessidades e desafios do ambiente econômico, tecnológico e institucional da cadeia; 4) discutir e estabelecer os objetivos da CPA, bem como as estratégias competitivas para atingi-los; 5) definir os objetivos e o escopo da coordenação da qualidade, bem como as características de qualidade a ser coordenadas; 6) adequar a ECQ/MCQ às especificidades da CPA em questão; 7) definir a capacitação mínima necessária que cada segmento da CPA deve ter para participar da fase de implantação da ECQ/ MCQ, tanto em termos de ações internas à empresa como de ações comuns à cadeia; e 8) gerar plano de implantação da ECQ e executá-lo junto à CPA, o que inclui a aplicação e gerenciamento do MCQ. A Figura 6 resume as principais etapas aqui descritas.

Uma vez realizadas essas atividades, espera-se que a CPA esteja preparada para iniciar o processo de implantação propriamente dito. Dada a heterogeneidade entre os agentes da CPA, em termos de conhecimento e de adoção de tecnologias e práticas de gestão da qualidade, sugere-se que a implantação da ECQ e seus elementos seja planejada segundo "estágios de maturidade", ou nível em que a ECQ se encontra, durante seu processo de implantação na CPA, em relação aos seguintes aspectos: a) escopo da ECQ: definido em termos da quantidade de segmentos da CPA e respectivos agentes que participam da implantação, bem como da quantidade

Figura 5: Ações do agente coordenador na ECQ.

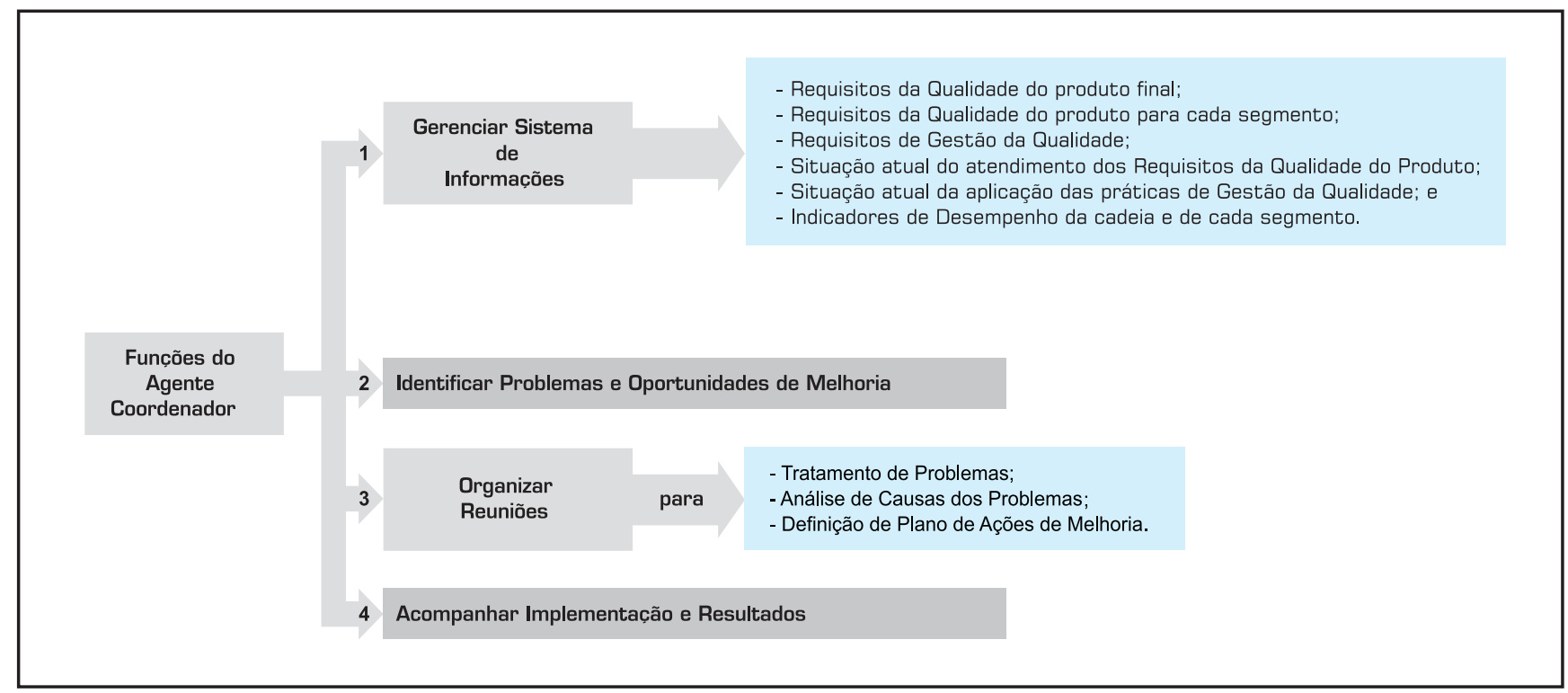

Fonte: Borrás (2005). 
de requisitos (RQP e RGQ) que são coordenados; b) nível de detalhamento das informações trocadas entre o agente coordenador e a CPA; c) nível de organização do agente coordenador (o quão desenvolvida está sua estrutura interna) e o grau de complexidade das atividades de coordenação da qualidade na CPA; d) nível de capacitação demandada, tanto para os segmentos e respectivos agentes da CPA como para o próprio agente coordenador; e e) módulos do MCQ que estão implantados no dia-a-dia da CPA.

Ainda durante o processo de implantação, é importante que os agentes participantes realizem, periodicamente, uma avaliação, indicando os pontos positivos e as dificuldades encontradas, de modo a construir um conjunto de lições aprendidas, as quais poderão ser proveitosas, seja para a melhoria da eficácia da implantação em andamento, seja como referência para outros processos de implantação da ECQ em outras cadeias.

Implantar a ECQ em uma CPA não é tarefa trivial, podendo-se prever muitas dificuldades, sobretudo aquelas derivadas do desnível existente entre os agentes (entre segmentos distintos ou mesmo dentro de um mesmo segmento da CPA) em termos de capacitação e da adoção de tecnologias e práticas de gestão da qualidade. Espera-se que os benefícios à CPA sejam crescentes à medida que: 1) aumente o tempo de uso do método por seus agentes; e 2) aumente o número de agentes da cadeia que se adequem às exigências mínimas necessárias para sua integração à ECQ e com possibilidade de uso do MCQ.

Com essas evoluções, os agentes da CPA passam a tratar a qualidade como fator estratégico para a cadeia e de vantagem competitiva junto ao mercado consumidor. A melhor compreensão da QDe e o comprometimento de todos os segmentos da cadeia em satisfazê-la reduziriam as perdas geradas pela não-qualidade, notadamente as geradas por falhas de produção, necessidade de retrabalho, subaproveitamento de recursos de transformação e a serem transformados. Como resultado, os agentes seriam capazes de identificar novos nichos de mercado, ao mesmo tempo em que aumentariam sua quota nos mercados nos quais a CPA já atua.

Com relação aos custos de implantação da ECQ e do MCQ, estes podem ser divididos em três categorias:1) custos

Figura 6: Fases e etapas do procedimento para implantação da ECQ/MCQ (PIEM).

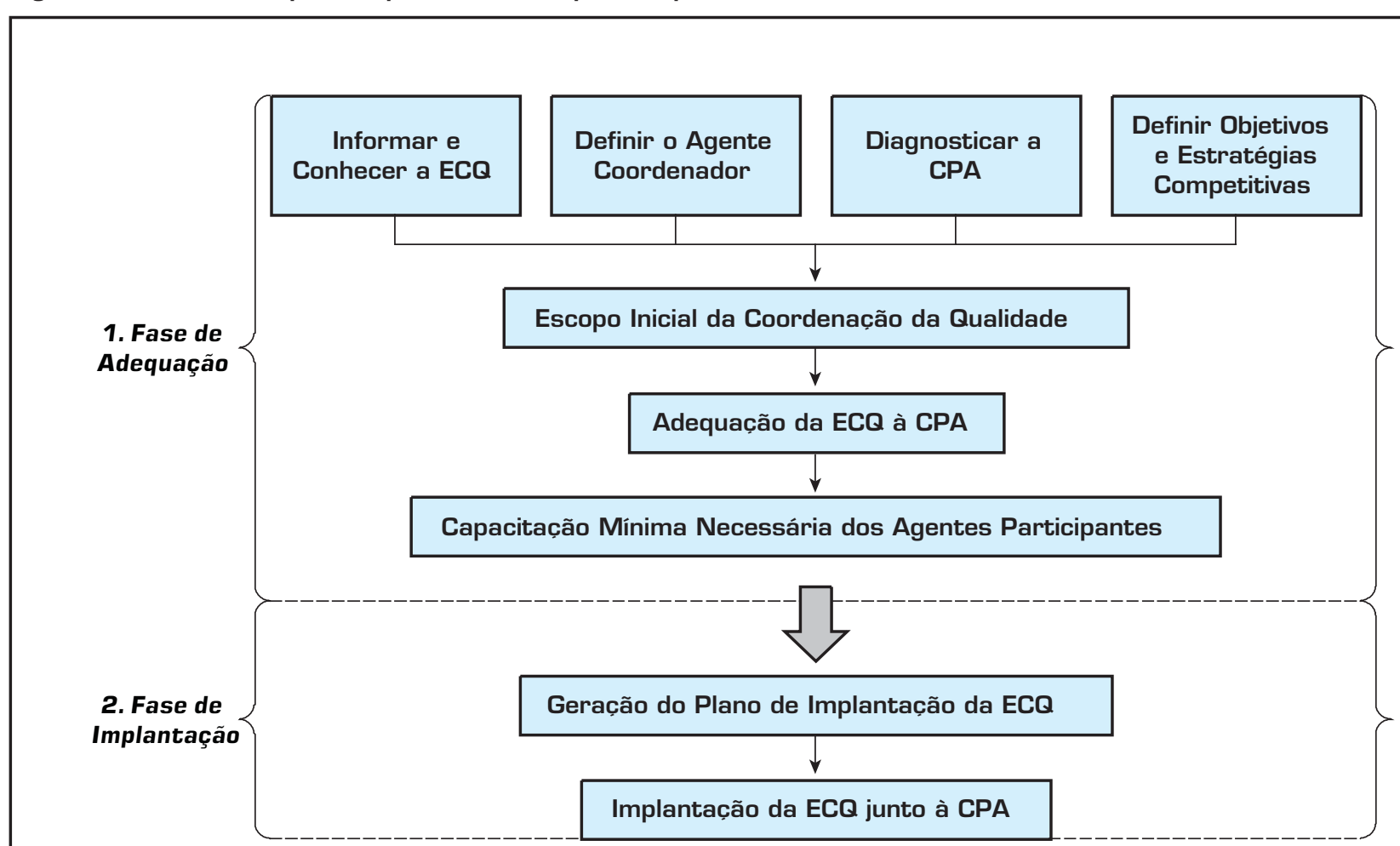

Fonte: Borrás (2005) e Borrás e Toledo (2006). 
de projeto e adequação da ECQ/MCQ à CPA; 2) custo de implantação da ECQ/MCQ; e 3) custo de operação da ECQ/ MCQ. Os custos iniciais de adaptação tecnológica e gerencial da CPA são amortizados ao longo do tempo.

$\mathrm{O}$ aprendizado organizacional reduzirá os custos de projeto e adequação e de implantação junto a novos agentes da cadeia, além de reduzir os custos de operação junto a todos os agentes envolvidos.

Na medida em que a ECQ/MCQ é utilizada, o incremento dos benefícios, gerado pela expansão da quota de mercado ou pela redução dos custos da não-qualidade, deverá compensar os custos de operação e manutenção.

\section{CONSIDERACÕES FINAIS}

Esta seção de considerações finais está dividida em duas partes: a primeira sobre aspectos relativos à forma e ilustração do MCQ e a segunda sobre aspectos relacionados à aplicabilidade da ECQ/MCQ.

\section{Quanto à forma e ilustração do MCQ}

O MCQ é constituído por módulos organizados de modo que a execução da ECQ/MCQ possa se dar de forma contínua, ou seja, a informação contida ou oriunda de um dado módulo, por exemplo o módulo 7 , depende da informação contida no módulo anterior, neste exemplo o módulo 6 que, por sua vez, é resultado das informações trabalhadas do módulo 1 ao módulo 5 .

O MCQ, então, é composto por "blocos" interdependentes, daí o porquê de cada bloco ser chamado de "módulo". O conjunto desses blocos ou módulos estrutura o MCQ. Alguns desses módulos são executados em etapas e, como discutido anteriormente, os módulos a ser implantados numa CPA dependem do momento ou circunstância em termos de gerência da produção na qual a cadeia se encontra.

Como discutido anteriormente, no momento de explicação do PIEM, cadeias num nível mais avançado de organização e gerenciamento da produção poderão ter a $\mathrm{ECQ}$ e uma maior quantidade de módulos do MCQ implantados, enquanto que numa CPA pouco ou mal organizada e cujos agentes se encontram num nível inicial de capacitação para gerenciamento da qualidade, poderá haver apenas o módulo 1 do MCQ implantado e seus agentes terão que submeter-se a um processo de aperfeiçoamento gerencial para integrar-se à ECQ.

A discussão detalhada sobre a aplicação da ECQ/MCQ encontra-se ilustrada em Borrás (2005). Por motivo de adequação do tamanho do texto aos padrões da Revista, tal ilustração, por ser extensa, não pôde ser incluída neste artigo.

\section{Quanto à aplicabilidade da ECQ e do MCQ}

O principal desafio para a coordenação da qualidade ao longo de uma CPA está na capacidade de estabelecer uma relação de cooperação entre os agentes da cadeia, a qual pode ser entendida como a reunião de empresas interdependentes que agem juntas para gerenciar os fluxos de produção, de serviços e de informações na cadeia, a fim de satisfazer o consumidor final e minimizar custos.

A proposta apresentada constitui uma forma de coordenação baseada numa concepção sistêmica da qualidade, em que se substitui e complementa o controle interno e individual de cada segmento por uma coordenação por meio de uma estrutura e de um método orientados para a prevenção de falhas e de perdas e, também, para a melhoria da qualidade ao longo da CPA. A estrutura e o método devem ser projetados como um mecanismo de incentivo e controle mais eficiente, para integrar e compartilhar informações e custos sobre a qualidade, contribuindo para a competitividade da cadeia. Busca-se gerar a cooperação e participação na garantia da qualidade de todos os envolvidos na cadeia de produção.

\section{uando há grande número de agentes, para realizar a função de agente coordenador.}

À medida que os padrões de qualidade tendem a ser definidos na própria cadeia ou de forma mista (público e privado), buscam-se mecanismos próprios intra-cadeia para acompanhamento e avaliação do atendimento a esses padrões, que é o sentido da proposta de estrutura de coordenação aqui apresentada.

Os atributos de qualidade e de gestão, que uma cadeia pretende sinalizar para o mercado, têm suas especificidades em função das categorias de produtos (bens de procura, de experiência ou de crença). Entretanto, acredita-se que a aplicação da ECQ/MCQ possa ser devidamente adequada para qualquer tipo de bem transacionado e de estrutura de governança dominante (mercado, mista ou hierárquica). Uma vez que as transações de bens de crença têm uma importância crescente no sistema agroalimentar, a ECQ e o MCQ podem ser utilizados para a redução de diferenças entre os atributos de qualidade sinalizados pela cadeia e os efetivamente realizados, incrementando a confiança do consumidor e a competitividade da cadeia.

A aplicação da ECQ/MCQ requer interação constante entre o agente coordenador, os segmentos da cadeia, instituições públicas e entidades de representação e de regulamentação e fiscalização. O envolvimento de diferentes tipos 
de organização pode dar à gestão da cadeia de produção um aspecto imparcial e de equilíbrio, entre os interesses das partes envolvidas na coordenação.

Outros modelos, publicados na bibliografia internacional, buscam apoiar a coordenação de cadeias de produção agroalimentares, como os apresentados por Trienekens, Beulens e Beek (2000), Lehnert, Schmitz e Petersen (2000), SchwarzBovee et al. (2000), Eroglu, Machleit e Davis (2001), McCarthy e Henson (2002), Poole et al. (2002), McDermott e Botha (2002), Jones e Zobel (2002). Porém, o que diferencia

semelhantes, ainda que não necessariamente implicando em se ter módulos com um mesmo conjunto de características, o MCQ apresenta o Módulo 8 de auto-avaliação e aperfeiçoamento, que possibilita indicar necessidades de melhoria do próprio método e da estrutura de coordenação. Além disso, a ECQ, o MCQ e o PIEM representam uma sistemática de aplicação que permite sua adequação a cadeias de diferentes estruturas e tipos de produção.

No entanto, reitera-se a expectativa de que a ECQ e o MCQ propostos contribuam para efetivamente garantir e, continuamente, melhorar a qualidade dos produtos ao longo da cadeia, com vistas à agregação de valor, à redução de perdas e de custos de produção e à segurança do alimento.

Para tanto, reforça-se a importância de que para a efetivação da

a proposta aqui apresentada em relação a tais modelos é o fato da ECQ/MCQ: a) focar a coordenação da qualidade; b) prever condicionar o agente coordenador como um elemento diferenciado na estrutura de coordenação, devendo ser capaz de compreender a CPA holisticamente e tomar decisões imparciais, visando o bem-estar da cadeia; c) apesar de todos os modelos apresentarem a informação como base para a coordenação da cadeia, a ECQ/MCQ prevê a participação de agentes de todos os segmentos da CPA no processo de transmissão, recebimento e gestão de informações advindas tanto dos ambientes mercadológico e institucional, quanto de outros segmentos da cadeia, incentivando a simetria informacional e o incremento da integração e participação dos segmentos nas tomadas de decisão da cadeia; d) é o único modelo que considera os requisitos da qualidade em amplo espectro, abarcando a problemática sociocultural e ecológica; e e) supõe o método inserido no contexto de uma estrutura de coordenação da cadeia. Apesar de os módulos dos referidos modelos e do MCQ apresentarem algumas funções estrutura e do método de coordenação e obtenção de seus benefícios, estejam consolidados: a) a organização da cadeia de produção agroalimentar e seus segmentos; b) a correta identificação dos requisitos de qualidade e de gestão da qualidade dos clientes e do ambiente institucional; c) o desdobramento dos requisitos da qualidade para os agentes participantes; d) o estabelecimento de um sistema de informações confiável e eficaz; e) a visão compartilhada de objetivos, indicadores de desempenho, problemas e planos de ação; e f) a atuação equilibrada e constante do agente coordenador com um perfil proativo e integrador dos interesses coletivos.

Estão sendo realizados estudos para viabilizar a implementação de tais estrutura e método em quatro cadeias de produção agroalimentar: açúcar refinado, vegetais minimamente processados, frango e mel. Todos esses estudos estão sendo viabilizados e iniciados a partir de convites de instituições públicas e privadas que se interessaram pelos benefícios que a ECQ/MCQ poderá trazer para as CPAs às quais pertencem.

Artigo recebido em 20/06/2007

Aprovado para publicação em 24/10/2007 


\section{- Referências}

BAINES, R. N.; DAVIES, W. P. Quality assurance in international food supply. In: INTERNATIONAL CONFERENCE ON CHAIN MANAGEMENT IN AGRIBUSINESS AND THE FOOD INDUSTRY, 3., 1998 Wageningen. Proceedings..., Wageningen Wageningen Academic Publishers, 1998 p. 213-223.

BERG, L. Trust in food in the age of mad cow disease: a comparative study of consumers' evaluation of food safety in Belgium, Britain and Norway. Appetite, $\mathrm{v}$. 42, p. 21-32, 2004.

BOEHLJE, M. et al. Observations on formation of food supply chains. In INTERNATIONAL CONFERENCE ON CHAIN MANAGEMENT IN AGRIBUSINESS AND THE FOOD INDUSTRY, 3., 1998, Wageningen. Proceedings..., Wageningen: Wageningen Academic Publishers, 1998. p. 393-403.

BORRÁs, M. A. A. Proposta de Estrutura e Método para Coordenação da Qualidade em cadeias de producão agroalimentares. 2005. 342p. Tese (Doutorado em Engenharia de Produção) - Programa de Pós-Graduação em Engenharia de Produção. Universidade Federal de São Carlos, São Carlos, 2005.

BORRÁS, M. A. A.; TOLEDO, J. C. de. A Coordenação de Cadeias Agroindustriais: garantindo a qualidade e competitividade no agronegócio. In: ZUIN, L. F.; QUEIROZ, T. R. (org.). Agronegócios: gestão e inovação. São Paulo: Saraiva, 2006. p. 21-56.
EROGLU, S. A; MACHLEIT, K. A.; DAVIS, L. M. Atmospheric qualities of online retailing: a conceptual model and implications. Journal of Business Research, v. 54 n. 2, p. $177-184,2001$

FROHLICH, M. T.; WESTBROOK, R. Arcs of integration: an international study of supply chain strategies. Journal of Operations Management, v. 19, n. 2, p.185-200, 2001

HEIM, D.; MUMFORD, E. The future of BSE from the global perspective. Meat Science, v. 70, n. 3, p. 555-562, 2005.

JONES, E.; ZOBEL, C. A decision support system for value-added production in the mid-atlantic wheat industry. In: INTERNATIONAL CONFERENCE ON INTERNATIONAL CONFERENCE ON
PARADOXES IN FOOD CHAINS AND NETWORKS, 5., 2002, Wageningen. Proceedings..., Wageningen: Wageningen Academic Publishers, 2002 p. 870-882.

LEHNERT, S.; SCHMITZ, T.; PETERSEN, $B$. Risk and weak point analysis in the range of chain-oriented data acquisition. In: INTERNATIONAL CONFERENCE ON CHAIN MANAGEMENT IN AGRIBUSINESS AND THE FOOD INDUSTRY, 4., 2000, Wageningen. Proceedings..., Wageningen: Wageningen Academic Publishers, 2000. p. $409-416$.

MCCARTHY, M.; HENSON, S. Risk reduction strategies employed to minimise perceived risk when purchasing beef.
In: INTERNATIONAL CONFERENCE ON PARADOXES IN FOOD CHAINS AND NETWORKS, 5., 2002, Wageningen. Proceedings..., Wageningen: Wageningen Academic Publishers, 2002. p. 201-213.

MCDERMOTT, A.; BOTHA, N. Antecedents of value network relationship designs: a conceptual model. In: INTERNATIONAL CONFERENCE ON PARADOXES IN FOOD CHAINS AND NETWORKS, 5., 2002, Wageningen. Proceedings..., Wageningen: Wageningen Academic Publishers, 2002. p. 709-719.

POOLE, N.; BHUPAL, D. S.; MARSHALL, F. LINTELO, D. Quality assurance initiative for peri-urban food production in India. In: INTERNATIONAL CONFERENCE ON PARADOXES IN FOOD CHAINS AND NETWORKS, 5., 2002, Wageningen. Proceedings..., Wageningen: Wageningen Academic Publishers, 2002. p. 298-314.

RAMASAMY, I. The risk of accidental transmission of transmissible spongiform encephalopathy: identification of emerging issues. Public Health: Journal of the Royal Institute of Public Health, v. 118 , p. $409-420,2004$

SCHIEFER, G. Environmental control for process improvement and process efficiency in supply chain management - the case of meat chain. International Journal of Production Economics, v. 78, n. 2, p. 197-206, 2002.
SCHWARZ-BOVEE, E, H G G STERRENBURG, P.; MEER, R. R. A. van deer.; OOSTEROM, E. W. Development and implementation of an early warning system for the animal production chain. In: INTERNATIONAL CONFERENCE ON CHAIN MANAGEMENT AGRIBUSINESS AND THE FOOD INDUSTRY, 4, 2000, Wageningen. Proceedings..., Wageningen: Wageningen Academic Publishers, 2000. p. 633-636.

TRIENEKENS, J. H.; BEULENS, A. J. M.; BEEK, P. van. Development of (re) design options in supply chains. In: INTERNATIONAL CONFERENCE ON CHAIN MANAGEMENT AGRIBUSINESS AND THE FOOD INDUSTRY, 4., 2000, Wageningen. Proceedings..., Wageningen: Wageningen Academic Publishers, 2000. p. 109-119.

ZIGGERS, G. W.; TRIENEKENS, J. Quality assurance in food and agribusiness supply chains: developing successful partnerships. International Journal of Production Economics, v. 60-61, n. 3, p. 271-279, 1999.

ZYLBERSZTAJN, D. Estruturas de governança e coordenação do agribusiness: uma aplicação da nova economia das instituições. 1995. 238p. Tese (LivreDocência em Administração) - Programa de Pós-Graduação em Engenharia de Produção. Universidade de São Paulo, São Paulo, 1995.

\section{- Sobre os autores}

\section{Miguel Angel Aires Borrás}

Engenharia de Produção - Universidade Federal de São Carlos, campus Sorocaba

Professor Adjunto

End.: Universidade Federal de São Carlos, campus Sorocaba. Av. Darci Carvalho Dafferner, 200 - Alto da Boa Vista

18085-850 - Sorocaba/SP

Tel./Fax: (15) 3218-1619

E-mail:maborras@dep.ufscar.br

\section{José Carlos de Toledo}

Departamento de Engenharia de Produção - Universidade Federal de São Carlos, campus São Carlos

Professor Titular

End.: Universidade Federal de São Carlos, Departamento de Engenharia de Produção. Via Washington Luis, km 235

C.P. 676 - 13565-905 - São Carlos/SP

Tel.: (16) 3351-8236 (r. 9215) - Fax: (16) 3351-8240.

E-mail: toledo@dep.ufscar.br 